\title{
TECHNOLOGICAL PROPERTIES OF COVERING MASSES OF ELECTRODE COATINGS
}

\author{
M.F. GNATENKO \\ Company «Velma» \\ 3 Kaunasskaya Str., 02160, Kiev, Ukraine.E-mail: mavel@ukrpost.ua
}

\begin{abstract}
In domestic and foreign publications the problems of quality of manufacture of coated electrodes are considered quite insufficiently. In the present work an attempt was made to consider the causes for insufficient stability of the process of molding the electrodes and to share a practical experience in their elimination. The recommendations on optimizing the coating process in the manufacture of electrodes were given. 4 Ref.
\end{abstract}

Keywords: manufacture of welding electrodes, covering masses, technological properties, optimization, granulometric composition, composition and properties of charge

The quality of manufacture of coated welding electrodes is determined mainly by technological properties of covering masses. The most significant indicator of the quality is the difference in thickness of coating. This indicator manifests itself at the stage of deposition of covering mass (coating) on rods in the process of their molding under the pressures on mass of up to $800 \mathrm{~kg} / \mathrm{cm}^{2}$ and at speeds of up to $400 \mathrm{~m} / \mathrm{min}$.

For $90 \%$ the stability of molding process, including the polythickness of coating, is determined by molding (plastic) properties of covering masses.

It is characteristic that in the publications on this problem there are no clear regularities about the influence of different parameters and characteristics of technological components on molding properties and, moreover, there are no clear practical recommendations for technologists and manufactures.

The aim of the work is to consider the causes of instability of the process of molding the electrodes and the possible ways of their elimination.

Technological properties of masses. They include:

- molding (plastic) properties of covering masses: pressure of molding (the lower the better at a sufficient resistance to dents), degree of stability of flows formation at the lead-in molding area and, accordingly, the stability of polythickness of coating deposition on rods at molding; the degree of mass (coating) adhesion to the rod at their contact in the molding chamber;

- properties of raw masses (coating), which characterize their tendency to processing (to rupture of coating in plane of mating the rods of electrodes after coming out from the molding head), to cleaning the coating for the electrode holder and the contact end, to sticking of electrodes, sticking to the frames, etc.;

(c) M.F. GNATENKO, 2016
- drying properties of covering masses: resistance of structure of covering masses to the passage of humidity (removal from inner layers); degree of softening the masses during heating at the initial stages of drying; tendency of masses to shrinkage and to micro/ macrocracks; to delamination from the rod surface (cracks and delamination reduce the strength of electrodes coating);

- strength properties of coating of ready electrodes.

Causes of low technological properties and ways of their optimization. The results of experiments, manufacturing experience and their analysis on the basis of the available scientific publications [1-4] allow stating that molding properties of covering masses are determined by:

- composition of the charge (properties and the amount of any components); degree of wetting and chemical interaction of charge powders with a liquid glass; strength characteristics of particles components, etc.;

- properties of liquid glass;

- volumetric granulometric composition of the charge;

- properties of plasticizer (including different additives, salts, surface-active agents, etc.);

- type of mixer for preparation of covering masses (considered in the work).

First of all, let us consider the most obvious factor of influence on molding properties of masses - granulometric composition of the charge (volumetric):

- maximum size of particles of the components should not exceed the thickness of the electrode coating. To be more precise, it should be $2-3$ times smaller, because during interaction $2-3$ particles in the 
thickness of the coating can lock the channel when there are many such particles in the charge;

- there should be a maximum density of packing the charge particles to prevent dilatency and pulsation;

- it is necessary to minimize the friction coefficient between the layers of flow during formation of the electrodes coating flow at the inlet to a die (forming bushing). This is achieved by reducing the middle fraction $(+0.063-0.16)$ in the charge to a minimum, i.e. the coarse fraction $(-0.16+0.355: 30-35 \%)$ amounts to the thickness of one layer (frame layer), and the fine fraction $(-0.063)$ in the layer thickness provides a maximum packing density and a minimum roughness of the layer;

- volume of fine fraction $(-0.063)$ of more than $65-70 \%$ should not be admitted in the charge, as a number of flow layers at the stage of formation of coating thickness increases sharply (by tens of times), and accordingly, a number of degrees of the layers shear also increases sharply. This results in unstable formation of flows in place and time (polythickness pulsation).

All the other problems associated with polythickness (causes and consequences) will be considered at the following condition:

- granulometric composition of the charge of covering masses is optimum and the same;

- purely chemical interaction of components with liquid glass is absent (including that with evolution of gaseous products).

Composition of charge and physicochemical properties of individual components are the following:

- presence of components with anisodiametrical shape of particles (mica, talc, vollostanit, kaolin, cellulose etc.) in a sufficient amount (not lower than 4-6\%) in the charge reduces the inner friction in the covering mass during formation of flows from the diameter of sleeve to the diameter of coating. It is also important that such particles reinforce the covering mass and, respectively, the coating, i.e. the resistance to dents, etc. increases. As a rule, such components are used in coatings of rutile, rutile-cellulose, ilmenite type, etc. The covering masses of such electrodes are characterized by good molding properties (ANO-4, OZS-4, MP3, ANO-13, ANO-36, etc.);

- presence of components in the charge which have the fracture strength on compression and shear being at the level of pressures to the mass during molding of electrodes of $300-800 \mathrm{~kg} / \mathrm{cm}^{2}$. It is known that this field covers strength characteristics of marble, magnesite, dolomite, etc. The most outstanding representatives of such charges (covering masses) are the electrodes of grades UONI 13/45, UONI 13/55, ANO-9, etc. In the composition of such charges there is ap- proximately $50 \%$ of marble with the fracture strength of about $500 \mathrm{~kg} / \mathrm{cm}^{2}$. The covering masses of such electrodes are characterized by low molding properties (and, in general, by technological ones). At their molding at the pressures higher than $400-500 \mathrm{~kg} / \mathrm{cm}^{2}$ (on mass) in the inlet area and in the molding chamber a partial destruction (spalling) of marble particles occurs with formation of new surfaces, which withdraw a part of moisture from the film of liquid glass gel. In those areas the mass thickens, the "crackers» are formed, the stability of mass flow formation is violated, the polythickness begins to pulsate in the direction and time. Also, frequently an increase in pressure up to locking the channels occurs.

The production experience gained earlier shows that, for example, the electrodes of grade ANO-4 are usually molded at pressures of $600-700 \mathrm{~kg} / \mathrm{cm}^{2}$ (on mass), and UONI 13/55 - at pressures of 350$450 \mathrm{~kg} / \mathrm{cm}^{2}$. In the first case, the mass contains much mica in its composition (24\%) and little relatively soft particles of magnesite (only $15 \%$ ). Electrodes ANO-4 can be molded at high pressures without violation of integrity of magnesite particles, especially in the presence of mica. In case of UONI 13/55 the effect is the opposite and it must be considered during molding, providing an increased stability of this process due to improvement of molding properties of masses using the appropriate methods of plasticizing; reducing the pressure of molding by using the effective molding of inlet areas and heads; application of intensive mixers for preparation of masses, as well as using the grade of marble with the strength in the range of $1000 \mathrm{~kg} / \mathrm{cm}^{2}$.

The application of flotation fluorite may influence on deterioration of molding properties of covering masses of electrode UONI 13/55 (etc.) of fluoride calcium type, which often contains particles of a relatively large amount of flotation reagents on the surface. This greatly reduces the wetting of fluorite with liquid glass. The degree of adhesion of liquid glass (gel) to fluorite particles decreases and the friction between the particles increases. The flow becomes more unstable, i.e. the degree of wetting of the charge particles determines the quality of coating of liquid glass and the level of friction between the particles during the flow increases. The molding process becomes less stable.

Role of liquid glass in the properties of covering masses. The technological, including molding, properties of covering masses depend by $70-80 \%$ on the type, characteristics and properties of liquid glass (its influence on welding and technological characteristics is not considered).

The main purpose of liquid glass in covering masses (from the viewpoint of molding) is to mini- 
mize friction between the particles of charge during formation of flow from the diameter of press cylinder to the diameter of electrodes coating at their molding and after molding the coating should have sufficient resistance against dents and mechanical damages. And now let us look how covering and reinforcing properties of liquid glass in a covering mass manifest themselves.

To establish the mechanisms and processes occurring during preparation of covering masses, it is necessary to divide this stage into two stages.

The first stage: distribution of liquid glass in charge, i.e. the coating of charge of particles with liquid glass. This stage should be carried out as quickly as possible. Moreover, it is necessary to provide a good wettability of surfaces of almost all the particles with liquid glass (by applying the corresponding special surfactants, if necessary). For preparation of masses the most effective are intensive mixers.

The second stage: formation of certain physical and mechanical properties of covering masses, as a consequence of reaction of liquid glass on the contact interaction with surface of particles of different components, such as:

- withdrawal of moisture from the film of liquid glass;

- adsorption processes (including interaction of electric potentials on the boundary surface);

- ion exchange between liquid glass and individual components;

- different influence of different types of plasticizers and special additives on liquid glass.

From work [2] it is known that as a result of such similar processes a gelling occurs in liquid glass (sharp increase in viscosity, polymerization, formation of organosilicon structures: gelling, coagulation). At the same time, depending on the characteristics of liquid glass, its parameters and influencing factors, the structure of liquid glass gel is produced different: soft, medium and hard.

To soft structure of the produced gel the liquid glasses are prone having a low modulus $(<2.9)$. To hard structure those are prone having a high modulus ( $>3.1$ ) and, of course, to medium one - those with modulus of $>2.9$ and $<3.1$. Let us see how it is manifested. If one takes a low modulus liquid glass on an index finger and rub it with a thumb, then its condition will not change for a long time. It will remain sticky (soft structure). If the same procedure will be done with a liquid glass of a high modulus, then very quickly a dry «sand» will form between the fingers (hard structure of the gel). And with a medium modulus, a layer of jelly type - the gel structure of medium hardness - will be formed between the fingers. In covering masses it is manifested during their preparation, i.e. if the mass is sticky, it «does not twist» for a long time, it is not stirred, then a gel structure is soft. If the mass becomes dry very quickly and requires an additional amount of liquid glass, if it moves hard between the fingers, crumbles, then a gel structure of liquid glass becomes hard. In a medium variant the mass becomes not sticky, but under an index finger and a thumb it moves well (even at repeated punching).

What is the cause of formation of different structures of liquid glass gel in the film of covering masses and their properties? In the process of liquid glass gelling, as is known, the processes of polymerization, gelling and coagulation of colloid particles of organosilicon compounds occur. As a result, both planar weak structures (soft), as well as volume structure (hard) may be formed depending on the modulus of liquid glass. At the medium modulus in the structure of a gel, both planar as well as three-dimensional structures in combination are present. At the moment of gelling (coagulation) the microformations (particles) have high adhesive and cohesive properties. As far as at the first stage the liquid glass has already covered the particles, at the second stage the gelling (coagulation) of film of liquid glass occurs, which is accompanied by a high adhesion, resulting in a quality coating of gel particles. It does provide the flow of covering masses at molding. So:

- at a low modulus the gelling is very weak (planar), adhesion to the particles is weak, the friction between the particles is high, the flow is unstable;

- at a high modulus the gelling is very intensive (coagulation), volumetric, the cohesion in the film of liquid glass is higher than the adhesion to the particles, so they are coated unevenly, the flow is not stable, and the coating «is loosened» as far as the mass in the structure is not restored (break of continuity). The flow in this case cannot be stable in the directions and time. The coating is poorly maintained on the rod. Apparently, the structure of such a gel has an elasticity associated with a porous structure, due to a large quantity of cohesive forces in microparticles of the gel. This is also in favor of the process of «loosening» the mass.

So, how do we control the gel structure? In case of a soft gel structure, it is necessary to harden it. In case of a hard structure, it should be soften bringing it to the medium level using the appropriate methods. In order to harden the gel structure from soft to medium it is necessary to apply an active plasticizer. This is potash, soda, technical CMC, etc. The electrolyte components cause activation of gelling process, as a result of which the medium gel structure is produced and the effect of increasing plastic (molding) proper- 
ties is achieved. It should be noted that there are also other methods for increasing the degree of gelling: increase in viscosity of the applied liquid glass, use of finer component which activates the gelling process in liquid glass more intensively, or applying the liquid glass with a higher modulus.

If the gel structure of liquid glass in the covering mass becomes hard, it is necessary to:

- reduce the viscosity of a liquid glass, or to exclude active plasticizers from the charge;

- apply the inactive plasticizers (purified CMC, pure electrode cellulose, etc.). Thus, CMC, «being dissolved» in the film of a liquid glass (with reduced viscosity), prevents the formation of a hard gel;

- it is necessary that the components actively influencing the process of gelling were applied with a larger granulometric composition;

- use additives of alkali solutions $(\mathrm{Na}, \mathrm{K})$ to decrease the modulus of liquid glass.

Some of the mentioned methods (both such as the first ones for soft, as well as the second ones for hard) can be realized already at the stage of preparation of covering masses (if the mass becomes soft, then active plasticizers should be added, and if dry, then the water and some purified CMC (EC) should be added.

The covering mass, like the charge-liquid glassplasticizer system considering the activity of individual components, should be prepared to produce a film of liquid glass gel of medium hardness structure.

In our opinion, the causes of poor plastic (molding) properties of covering masses and the ways of their improvement (optimization) were defined.

\section{Conclusions}

1. Granulometric composition of the charge (volumetric) should be the following: with the maximum density of packing, otherwise dilatency (pulsation and squeezing of liquid phase) will occur; with the minimum roughness of the layer flowing-sliding and the minimum pressure of molding (minimum content of medium fraction); with a minimum number of layers of up to three at the formation of mass flow reaching the thickness of coating (amount of soft fraction in the charge of more than $65-70 \%$ should not be admitted, otherwise, a strong pulsation will occur).

2. A high content of carbonates in the charge (electrodes UONI, ANO-9, etc.) negatively influences the plastic properties of the masses, as far as their strength is low (about $500 \mathrm{~kg} / \mathrm{cm}^{2}$ ) and at the pressures of molding on the masses the fracture of particles of such components (erasing) occurs, which causes the formation of crackers, violation of the formation of flows and pulsation.

It is required to apply the effective methods of plasticizing in order that the pressure of molding does not exceed about $500 \mathrm{~kg} / \mathrm{cm}^{2}$ (on the mass); to minimize the pressure losses in the inlet and molding areas; to use the grades of high-strength carbonates (including marble of $1000 \mathrm{~kg} / \mathrm{cm}^{2}$ strength); to produce electrodes of the corresponding grades with a small amount of carbonates.

3. To use a liquid glass with modulus of $3 \pm 1$ and the corresponding plasticizers, depending on the activity of a charge. It is also possible to use the high modulus liquid glasses, but of a very low viscosity and only in combination with a high-quality purified CMC. In all the cases it is necessary to try achieving the structure of medium hardness of the gel, which provides the highest plastic properties of masses.

1. Vornovitsky, I.N. (2001) Quality control of welding electrodes during their manufacture. Moscow: IKAR.

2. Ignatkina, O.I., Lukianova, O.I., Borsuk, P.A. et al. (1999) Rheological properties of gels formed in interaction of liquid glass and hardeners of complex ether type. Lit. Proizvodstvo, 10, 39-41.

3. Marchenko, A.E., Gnatenko, M.F. (1980) Peculiarities of flow of electrode covering masses detected by capillary plastometer. In: Information of CMEA on Development of scientific principles and new technological processes of welding, surfacing and thermal cutting of materials and alloys for manufacturing of welded structures and efficient welding consumables and equipment, Issue 1, 106-117.

4. Marchenko, A.E. (2016) Rheological investigations of non-isothermal pressure flows of coating mixtures for welding electrodes. The Paton Welding J., 1, 17-29. 